\title{
Normas generales de presentación y publicación Revista Iberoamericana de Estudios Municipales
}

\section{Alcance y política editorial}

RIEM - Revista Iberoamericana de Estudios Municipales tiene como objetivo difundir la investigación relacionada con el quehacer municipal, en el ámbito académico nacional y extranjero.

Los trabajos que se publican son inéditos y de investigación, provenientes de las diversas áreas de investigación relacionadas con el mundo municipal, y su quehacer en el espacio local. Se publican prioritariamente artículos que se fundamentan en investigaciones producto de proyectos Fondecyt (Conicyt) o equivalentes (por ejemplo, proyectos de investigación interna universitaria u otros). Los artículos deben ceñirse estrictamente a las Normas de Publicación de la Revista.

La evaluación de los artículos es llevada a cabo por pares externos nacionales y extranjeros, quienes consideran aspectos como la originalidad de la investigación o manuscrito y la relevancia para el conocimiento científico de la investigación municipal y sus diversas áreas de acción, como salud, educación, seguridad, uso de tecnología, entre otras.

\section{Tipos de Publicación}

La Revista Iberoamericana de Estudios Municipales (RIEM), acepta trabajos originales o inéditos, principalmente de investigación:

a. Artículos o Avances de Investigación: trabajos que presenten avances o resultados de investigaciones, indicando fecha de inicio y finalización de la investigación y las entidades ejecutoras.

b. Artículos de Reflexión: aquellos trabajos que analicen o interpreten, algún tema de elección libre, inscritos en una temática específica.

c. Reseñas o recensiones bibliográficas: revisiones de libros y artículos. 


\section{Normas de Presentación}

La Revista Iberoamericana de Estudios Municipales recibe colaboraciones para su publicación de acuerdo con las siguientes normas:

a. Los textos deberán ser originales e inéditos.

b. Se recibirán trabajos en español, portugués e inglés.

c. Los Artículos de Investigación y Reflexión, deberán tener una extensión mínima de 9.000 y máxima de 10.000 palabras (incluyendo título, tablas, figuras y referencias bibliográficas.

d. Las Reseñas o Recensiones Bibliográficas, deberán tener una extensión mínima de 1.000 y máxima de 2.000 palabras, incluyendo título, tablas, figuras y referencias bibliográficas).

e. Se deberá anteponer 2 abstract del trabajo (español, inglés o portugués), de no más de 120 palabras cada uno y cinco palabras claves en ambos idiomas.

f. En un documento separado, deberá incluirse una biografía breve de cada uno de los autores que no exceda de 100 palabras (incluyendo sus grados académicos más relevantes, las instituciones académicas en las cuales se cursaron y cargos actuales). No debe olvidarse de incluir: nombre completo del/os autor/ es; filiación institucional, correo postal, correo electrónico, teléfono, fax, ciudad y país.

\section{Evaluación}

La Revista Iberoamericana de Estudios Municipales informará a cada autor del recibo del artículo enviado.

El Editor decidirá sobre el envío de los artículos recibidos a pares evaluadores para su revisión. Todos los artículos serán sometidos a arbitraje por parte de dos evaluadores independientes (evaluación ciega), especializados en el área. En caso de disidencia, será el Editor quien decida sobre la evaluación del artículo. La evaluación será comunicada vía correo electrónico a los autores, en un plazo no superior a 40 días como máximo desde el cierre de la convocatoria. Por último, el Editor informará al autor, si el artículo fue aprobado, aprobado con recomendaciones o rechazado para su publicación.

Aquellos autores cuyos artículos o notas de investigación sean publicados, recibirán 2 copias del ejemplar de la revista donde éste apareció. Además

Ante cualquier consulta sobre la presentación de artículos o evaluación de los mismos, pueden consultar al Editor:

Sr.Andrés Chacón Romero.

revista@ichem.cl 


\section{Normas Básicas de escritura (según la A.P.A.)}

a) Niveles de títulos: el cuerpo del texto deberá ser subdividido mediante los siguientes niveles de títulos:

- Principal, en negrita, centrado y caja alta (mayúsculas)

- Secundario, en cursiva, margen izquierdo y caja alta

- Terciario, en cursiva, margen izquierdo, caja baja y el texto en la misma línea

b) Alineación: el texto deberá estar alineado a la izquierda, exceptuando las citas bibliográficas de más de 40 palabras.

c) Palabras en negrita: No se utilizan. Para enfatizar se utilizan las cursivas en los siguientes casos:

- Palabras en otros idiomas (incluido el latín). En este caso, también se pueden utilizar las comillas

- Símbolos estadísticos y palabras algebraicas

- Palabras, letras o frases que aparecen mencionados como símbolos o ejemplos

- Transcripciones de registros orales

d) Extensión de los párrafos: Cada párrafo no podrá tener menos de tres oraciones ni más de ocho. Ello ayuda a la mejor compresión del texto. Lo mismo ocurre con las oraciones: ellas no podrán exceder las tres líneas.

e) Mayúsculas o caja alta: No se utilizarán las mayúsculas en ninguno de los siguientes casos:

- Para nombrar autores en el cuerpo del texto

- Para nombrar autores en la lista de Referencias

- Para enfatizar palabras dentro del texto

f) Siglas: Cuando se hace mención por primera vez en el texto a una sigla, deberá indicarse el nombre completo de la institución y entre paréntesis la sigla. Posteriormente, podrá utilizarse la sigla en reemplazo del nombre completo de la institución.

g) Abreviaturas: Las abreviaturas que se utilizarán en el texto deben explicarse al inicio de éste en un índice especial para ello. La primera vez que se utilice la abreviatura en el texto, deberá indicarse la palabra completa y entre paréntesis la abreviatura. En lo que siga, se podrá utilizar solamente la abreviatura. 
h) Expresiones numéricas:

Guarismos: se utilizan números en los siguientes casos:

- En números de 10 y mayores ( 15 hombres)

- En números menos de 10 que expresen comparaciones en la misma frase con números mayores (5 de cada 10 alumnos)

- En unidades de medida (el tamaño es de $12 \times 14$ )

- En las funciones estadísticas (ler. Cuartil)

- En los números que representan fechas, tamaños, tiempo, cantidades porcentuales, monedas, etc. ( 3 años, 2 semanas, Ih 34 mn, el 30 de marzo de 1994)

En palabras: escriben los números en letras en los siguientes casos:

- En números menores de 10 (cinco ensayos, ocho veces)

- En los números cero y uno

- Al principio de una oración (Quince navegantes)

- En fracciones (Un tercio de los consultados)

- En fechas o acontecimientos históricos (Dieciocho de Septiembre, Los Diez Mandamientos)

i) Pie de página: Los pie de página sólo se utilizan para la elaboración de citas explicativas. Se ubica un número superíndice en la palabra a aclarar y en la parte inferior de la página se efectúa la aclaratoria. La numeración debe hacerse en forma ascendente y consecutiva. Los pie de página no se utilizan para efectuar citas bibliográficas.

Tablas, gráficos, figuras e ilustraciones

- Cada tabla, gráfico, figura y/o ilustración que el autor utilice en el cuerpo del texto deberá estar indexada en una página exclusiva para ello.

- A cada tabla, gráfico, figura y/o ilustración deberá asignársele numeración consecutiva, de acuerdo al orden en que aparezcan en el trabajo.

- Al inicio de cada tabla, gráfico, figura y/o ilustración deberá llevar un título que la identifique. Si la tabla, gráfico, figura y/o ilustración no son propiedad del autor o de los autores, deberá indicarse la referencia. 


\section{Referencias}

Libros: Autor (apellido -sólo la primera letra en mayúscula-, coma, inicial del primer nombre (y del segundo, si lo hay), punto. En caso de varios autores, éstos se separan con una coma y antes del último con una " $y$ ". Año de publicación entre paréntesis, punto. Título completo de la obra en cursiva, punto. Ciudad de edición, dos puntos, editorial.

\section{Coulon, A. ( 1 988). La etnometodología. Madrid: Cátedra}

Capítulos de libros colectivos o actas: Autores y año (como se indicó anteriormente), título del capítulo, punto. Luego la palabra “En”, nombre de los autores del libro (inicial, punto, apellido); (Eds.) o (Dirs.) o (Comps).Título del libro en cursiva, páginas que ocupa el capítulo entre paréntesis, punto. Ciudad de edición, dos puntos, editorial.

Singer, M. (1 994). Discourse Inference processes. En M. Gernsbacher (Ed.), Handbook of Psycholinguistics (p.p. 459-5I6). New York:Academic Press.

Artículos de revistas: Autores y año (como en los casos anteriores). Título del artículo, punto. Nombre completo de la revista en cursiva y coma., volumen en cursiva, número de la revista entre paréntesis y pegado al volumen, coma, página inicial, guión, página final, punto.

Gutiérrez Calvo, M. y Eysenck, M.W. (1995). Sesgo interpretativo en la ansiedad de evaluación. Ansiedad y Stress, I (I), 5-20.

Referencias de información contenidas en la web:

- Artículo de revista consultado en versión web pero con edición impresa y electrónica idénticas (en general, están en formato PDF):

Vandenbos, G., Knapp, S. y Doe, J. (200I). Role of reference elements in the selection of resources by psychology undergraduates [Versión electrónica]. Journal of Bibliographic Research, 5, I I7-123.

- Artículo de revista consultado en versión web pero con edición impresa y electrónica parecidas pero no idénticas:

Vandenbos, G., Knapp, S. y Doe, J. (200I). Role of reference elements in the selection of resources by psychology undergraduates. Journal of Bibliographic Research, 5, I 17-I23. Recuperada I3 de Octubre de 200 I, de http://jbr.org/articles. html 
- Artículo en e-journal (sin edición impresa):

Fredrickson, B. L. (2000, 7 de Marzo). Cultivating positive emotions to optimize health and well-being.Prevention \& Treatment, 3,Artículo 000 la. Recuperado 20 de Noviembre de 2000, de http://journals.apa.org/prevention/volume3/ pre003000la.html

En este ejemplo, la primera fecha es la de publicación del artículo en la red, y la segunda es la de su recuperación de la red por parte del lector.

- Documentos sin autor conocido (poner título del documento) ni fecha (poner s.f., sin fecha):

GVU's 8th WWW user survey (s.f.). Recuperado 8 de Agosto de 2000, de http:// www.cc.gatech.edu/gvu/usersurveys/surveyl997-10/

- Documentos disponibles en la web de una universidad do departamento:

Chou, L., McClintock, R., Moretti, F. y Nix, D. H. (1 993). Technology and education: New wine in new bottles: Choosing pasts and imagining educational futures. Recuperado 24 de Agosto de 2000, de Columbia University, sitio web del Institute for Learning Technologies: http: //www.ilt.columbia.edu/publications/ papers/newwinel.html

- Tesis:

Mendoza, L.E. (1969). La orientación como técnica pedagógica. Su aplicación en Panamá. Tesis doctoral no publicada. Universidad Complutense, Facultad de Filosofía y Ciencias de la Educación, Madrid.

- Diccionarios:

Real Academia Española (1992). Diccionario de la lengua española (tms. I y II) (2I Edición). Madrid: España

- Enciclopedias:

Planeta (Ed.) (1981). Nueva Enciclopedia Larousse (Vols. I-10). Barcelona, España: Autor 
Diarios y periódicos:

- Artículos con autor:

Sandoval,W. (2003, 28 de noviembre). Feria del Software en Venezuela. El Nacional, p.A 18

- Artículos sin autor:

Via Crucis por la paz y la libertad. (2004, 10 de abril). El Nacional, p. C 8

Editorial:

Una gran gesta ciudadana. [Editorial] $(2003,28$ de noviembre). El nacional, p. 10

- Papers de congresos, conferencias, seminarios, etc:

-

Pérez Gómez, Angel (1992). La formación del profesor como intelectual. Simposio Internacional sobre Teoría crítica e Investigación Acción, Valladolid, I-4 abril, (paper).

\section{Comunicaciones}

Las colaboraciones deben ser remitidos a Andrés Chacón Romero, Editor de Revista Iberoamericana de Estudios Municipales.

Coordinación Editorial:Verónica Candia Durán

Correo Electrónico: revista@ichem.cl

Teléfonos: (56-2) 8857743

Dirección: Gaspar Banda 38I0. San Miguel R.M.

WEB: www.ichem.cl 


\section{General rules for publication and presentation Iberoamerican Magazine of Municipal Studies}

\section{Scope and editorial policy}

RIEM - The Iberoamerican Magazine of Municipal Studies aims to spread the investigation related to municipal work in domestic and foreign academic scope.

Papers that are published are unreleased researches from various areas of investigation related to the municipal world, and its work in the local area. Published articles are mainly those that are primarily based on researches that are a result of Fondecyt projects (Conicyt) or equivalent (e.g., university internal research projects or other). The articles must strictly adhere to the Rules for Publication of the Magazine.

The assessment of the articles is carried out by external peers both national and foreign, who consider aspects such as the originality of the research or manuscript and the relevance to scientific knowledge of municipal research and various areas of action, such as health, education, security, and use of technology, among others.

\section{Types of Publication}

The Iberoamerican Magazine of Municipal Studies (RIEM) accepts original or unpublished works mainly of research:

a. Articles or Research Advances: papers that show progress or results of research, indicating date of beginning and completion of the investigation and the executing agencies.

b Discussion Articles: those who analyze or interpret some free choice issue inscribed on a specific theme.

c. Reviews or bibliographic reviews: books and articles reviews.

\section{Presentation Standards}

The Iberoamerican Magazine of Municipal Studies (RIEM) receives collaborations for its publication in accordance with the following rules:

a. The texts must be original and unpublished.

b. The articles will be received in Spanish, English and Portuguese.

c. The articles for research and discussion must have a minimum extension of 9,000 and maximum of 10,000 words (including title, tables, figures and references).

d. Book Reviews must have a minimum extension of 1.000 and maximum of 2.000 words. (Including titles, tables, figures and references). 
e. $\quad 2$ abstract should precede the article (in Spanish, English or Portuguese), not more than 120 words each and five key words in both languages.

f. In a separate document, it should be included a brief biography of each of the authors that does not exceed 100 words (including their most relevant academic degrees, occupations and current occupations, and a list of major publications.) You must remember to include: full name of the author(s), institutional affiliation, mailing address, e-mail, phone, fax, city and country. Also, in case of more than one author, indicate who is the person designated for the electronic communication.

g. Presentation standards under APA rule

\section{Evaluation}

The Iberoamerican Magazine of Municipal Studies (RIEM) will inform receipt of articles to each author.

The Editor will decide on sending the articles received for review to peers reviewers review. All articles will be subject to arbitration by two independent reviewers (blind evaluation), specialized in the area. In case of dissent, it will be the Editor who will decide on the evaluation of the article. The evaluation will be sent to authors, via email, not later than $\mathbf{4 0}$ days from the end of the call. Finally, the Editor will inform the author if the article was approved, approved with recommendations or rejected for publication

Those authors whose articles or research notes are published will receive 5 copies of the journal issue where it was published.

Should you have any questions about articles' submission or their evaluation, you may seek advice from the Editor:

Mr.Andrés Chacón Romero.

revista@ichem.cl

\section{Communications}

Contributions should be sent to Andrés Chacón Romero, Editor of "Revista Iberoamericana de Estudios Municipales”.

Editorial Coordinator:Verónica Candia Durán

Email: revista@ichem.cl

Telephone: (56-2) 8857743

Address: Gaspar Banda 3810. San Miguel R.M.

WEB: www.ichem.cl 


\section{Normas gerais de publicação e apresentação Revista Ibera americana de Estudos Municipais}

\section{Alcance e política editorial}

RIEM - Revista Ibera americana de Estudos Municipais tem como objetivo difundir a investigação relacionada com o que fazer municipal no âmbito acadêmico nacional e estrangeiro.

Os trabalhos que se publicam são inéditos e de investigação, provenientes das diversas áreas de investigação relacionadas com o mundo municipal, e seu que fazer no espaço local. Publicam-se prioritariamente artículos que se fundamentam em investigações produto de provectos Fondecyt (Conicyt) ou equivalentes (por exemplo, projetos de investigação interna universitária ou outros). Os artigos devem adequar se estritamente ás Normas de Publicação da Revista.

A avaliação dos artigos é levada a cabo por pares externos nacionais e estrangeiros, quem consideram aspectos como a originalidade da investigação ou manuscrito e a relevância para o conhecimento científico da investigação municipal e suas diversas áreas de ação, como saúde, educação, segurança, uso de tecnologia, entre outras.

\section{Tipos de Publicação}

A Revista Ibero americana de Estudos Municipais (RIEM), aceita trabalhos originais ou inéditos, principalmente de investigação:

a. Artigos ou Avances de Investigação: trabalhos que apresentem avances ou resultados de investigações, indicando data de inicio e finalização da investigação e as entidades executoras.

b. Artículos de Reflexão: aqueles trabalhos que analisem ou interpretem, algum tema de eleição livre, inscritos numa temática específica.

c. Indicações bibliográficas: revisões de livros e artigos.

\section{Normas de Apresentação}

A Revista Ibera americana de Estudos Municipais recebe colaborações para sua publicação de acordo com as seguintes normas:

a. Os textos deverão ser originais e inéditos.

b. Se receberão trabalhos em espanhol, português e inglês.

c. Os Artículos de Investigação e Reflexão, deverão ter uma extensão mínima de 9.000 e máxima de 10.000 palavras (incluindo título, tábuas, figuras e referencias bibliográficas. 
d. As Indicações Bibliográficas, deverão ter uma extensão mínima de 1.000 e máxima de 2.000 palavras. (incluindo título, tabuas, figuras e referencias bibliográficas).

e. Se deverá antepor 2 abstract do trabalho (espanhol, inglês ou português), de não mais de 120 palavras cada um e cinco palavras chave em ambos os idiomas.

f. Num documento separado, deverá incluir se uma biografia breve de cada um dos autores que não exceda de 100 palavras (incluindo seus graus acadêmicos mais relevantes, ocupações e cargos atuais, e um listado de publicações principais). Não deve esquecer-se de incluir: nome completo do/os autor/s; filiação institucional, correio postal, correio eletrônico, telefone, fax, cidade e país. Assim mesmo, em caso de ser mais de um autor, se deverá indicar quem será a pessoa designada para a comunicação eletrônica.

g. Normas de apresentação sob norma A.P.A.

\section{Avaliação}

A Revista Ibera americana de Estudos Municipais informará a cada autor do recibo do artigo enviado.

O Editor decidirá sobre o envio dos artigos recebidos a pares avaliadores para sua revisão. Todos os artigos serão submetidos a arbitragem por parte dos avaliadores independentes (avaliação cega), especializados na área, em caso de dissidência, será o Editor quem decida sobre a avaliação do artículo.A avaliação será comunicada via correio eletrônico aos autores, num prazo não superior a 40 dias como máximo desde o fechamento da convocatória. Por último, o Editor informará ao autor, si o artigo foi aprovado, aprovado com recomendações ou rejeitado para sua publicação.

Aqueles autores cujos artículos ou notas de investigação sejam publicados, receberão 5 copias do exemplar da revista onde este apareceu.

Ante qualquer consulta sobre a apresentação de artículos ou avaliação dos mesmos, podem consultar ao Editor:

\section{Sr.Andrés Chacón Romero.}

revista@ichem.cl

\section{Comunicações}

As colaborações devem ser remitidas a Andrés Chacón Romero, Editor de Revista Ibero americana de Estudos Municipais.

Coordenação Editorial:Verônica Candia Durán

Correio Eletrônico: revista@ichem.cl

Telefones: (56-2) 8857743

Endereço: Gaspar Banda 3810. São Miguel R.M.

WEB: www.ichem.cl 


\section{Autorización de publicación e inclusión en catálogos, bases de datos o índices bibliográficos y cesión}

Al momento de enviar las contribuciones, los autores/as declaran su autoría respecto del artículo enviado, y se autoriza a la Universidad Autónoma de Chile a publicar en la Revista “Iberoamericana de Estudios Municipales”, a su cargo, el trabajo/ artículo/paper/informe respectivo, tanto en su versión impresa o digital, o ambas, para la inscripción en el registro de propiedad intelectual, publicación y distribución. La autorización se concede a título gratuito, no siendo remunerado en forma alguna por el aporte académico en comento, como asimismo la autoría respecto del mismo, en conformidad con la ley $\mathrm{N}^{\circ} 17.336$ del 02 de octubre del año 1972.

Se autoriza expresamente y en forma gratuita a la Universidad Autónoma de Chile a que dicho trabajo/artículo/paper/informe, en forma total o parcial, individualmente o en conjunto con el resto del contenido de la Revista "Iberoamericana de Estudios Municipales", sea incluido en ediciones especiales futuras de la misma Revista o en catálogos, bases de datos o índices bibliográficos, impresos o digitales, sean éstos de libre acceso al público o sujeto al pago de derechos. 